\title{
Enterococcus faecalis endocarditis following percutaneous manipulation of a biliary tract calculus and ERCP
}

Allan R Ronald, MD, ANDREW LS PattulLo, MD

ABSTRACT: A case of Enterococcus faecalis endocarditis followed endoscopic retrograde cholangiopancreatography and percutaneous extraction of a biliary calculus is reported. The most likely cause of endocarditis, though unproven, is the latter procedure, as the bile is often infected during biliary tract obstruction, and bacteremia is frequent during percutaneous manipulations. Initial therapy with vancomycin was unsuccessful in clearing the bacteremia, possibly due to vancomycin tolerance of the isolate and lack of an aminoglycoside in the initial regimen. Cure was obtained when therapy with ampicillin and gentamicin was undertaken. Can J Infect Dis 1990;1(4):143-145

Key Words: Biliary, Endocarditis, Enterococcus faecalis, Endoscopic retrograde cholangiopancreatography, Vancomycin
B ACTERIAL ENDOCARDITIS OF A NORMAL HEART VALVE has not been reported as a consequence of $\mathrm{T}$ tube manipulation or endoscopic retrograde cholangiopancreatography (ERCP). The authors recently managed a case of Enterococcus faecalis endocarditis that occurred following these procedures, and which failed to resolve with initial therapy using vancomycin alone.

Department of Infectious Diseases and Medical Microbiology. Health Sciences Center Hospital, Winnipeg. Manitoba

Correspondence and reprints: Dr AR Ronald, 5th floor, Basic Medical Sciences Building. 730 William Avenue, Winnipeg, Manitoba, R3E OW3

Received for publication April 30, 1990. Accepted August 20,1990

\section{CASE PRESENTATION}

A 58-year-old male was admitted to hospital with a two week history of fever, chills and sweats. Six months previously he had had a cholecystectomy for acute obstructive jaundice, at which time calculi that could not be extracted were present in the common bile duct. He underwent ERCP twice in the next two months with removal of one calculus on each occasion. One week after the last ERCP and four months prior to admission, the T tube was cannulated and a final calculus extracted with difficulty. Prophylaxis with cefoxitin was used at the initial surgery, and a bile culture at surgery was negative. The subsequent procedures were done without antibiotic prophylaxis, and no cultures were taken. 
Two months prior to admission the patient had an episode of vertigo with numbness of the left hand and several episodes of diplopia. Following this he experienced migratory arthralgia, localized swelling of the dorsum of the right hand and pain in the right calf lasting several days. Two weeks before admission, fever, chills and sweats began.

On admission blood pressure was 90/50 mmHg. The apical impulse was hyperdynamic and located in the sixth left interspace. Heart sounds were normal, and a grade $3 / 6$, high pitched, early diastolic murmur was heard radiating throughout the precordium. No signs of heart failure nor any peripheral stigmata of endocarditis were found. The white blood cell count was $9.5 \times 10^{9} / \mathrm{L}$, and the erythrocyte sedimentation rate was $69 \mathrm{~mm} / \mathrm{h}$. Blood cultures grew E faecalis. Urinalysis was unremarkable and a urine culture was negative. Chest roentgenographic examination showed an increased heart diameter.

Echocardiography revealed normal mitral and tricuspid valves with mild mitral insufficiency. A large, mobile, echogenic mass was present in the left ventricular outflow tract, attached to the right coronary cusp of the aortic valve. Moderately severe aortic insufficiency and a small volume of pericardial fluid were also noted.

The patient recalled that he had had hives after penicillin therapy in the past, so treatment with intravenous vancomycin $500 \mathrm{mg}$ every $6 \mathrm{~h}$ was begun. Trough vancomycin levels of 13.2 and 14.5 $\mathrm{mg} / \mathrm{L}$ were measured on the second and sixth days of therapy, respectively. After five days of therapy, repeat blood cultures again grew $E$ faecalis. The patient was skin-tested for penicillin sensitivity with negative results and therapy was changed to intravenous ampicillin 2 g every $4 \mathrm{~h}$ with intravenous gentamicin $80 \mathrm{mg}$ every $12 \mathrm{~h}$.

Susceptibility testing showed the E faecalis to be susceptible to ampicillin. The minimal inhibitory concentration (MIC) and minimal bactericidal concentration (MBC) for ampicillin were both $1.0 \mathrm{mg} / \mathrm{L}$. The MIC and MBC for gentamicin were both $16.0 \mathrm{mg} / \mathrm{L}$. The organism was also sensitive to vancomycin with an MIC of $1.0 \mathrm{mg} / \mathrm{L}$, but the MBC was greater than $64 \mathrm{mg} / \mathrm{L}$. A peak serum cidal titre was $1 / 256$ while the patient was on intravenous ampicillin and gentamicin.

The patient's symptoms resolved gradually on treatment. After six weeks of intravenous antibiotics, therapy was switched to oral amoxicillin $1 \mathrm{~g}$ every $8 \mathrm{~h}$ for a further six weeks. Renal function remained normal throughout treatment, and after two months the sedimentation rate and white blood cell count were normal. There have been no significant complications during the one year since completion of treatment.

\section{DISCUSSION}

The diagnosis of bacterial endocarditis is substantiated in this patient by a typical clinical course suggestive of cerebral and peripheral emboli, as well as persistently positive blood cultures and the unequivocal echocardiogram. Group D streptococci (including enterococci) are frequently the cause of endocarditis (1). That the organism originated in the patient's obstructed biliary tract has not been proven, as a culture of bile was not obtained during the $T$ tube manipulation. However, $E$ faecalis is frequently cultured from the biliary tract when obstruction or cholangitis is present, and is a common cause of bacteremia during biliary tract manipulative procedures (2).

In this patient the initial operation was not likely to have resulted in bacteremia as the bile was sterile at surgery. The three procedures that followed are each a more likely source of bacteremia as no prophylaxis was used, and there was persistent obstruction of the common bile duct with stones for an extended period. In this setting, infection of the biliary tract is frequent. In 28 patients, bacteriobilia was found in 31 of 32 aspirates taken through an indwelling $\mathrm{T}$ tube at the time of percutaneous extraction of calculi. Enterococcus species made up 17\% of bile isolates (2).

ERCP could have been associated with subsequent infection of the aortic valve; however, this procedure is associated with bacteremia infrequently. Low et al (3) found bacteremia associated with ERCP and sphincterotomy in only 3\% of 95 procedures. In another study, Low et al (4) found no episodes of bacteremia during 101 ERCP procedures. Dutta et al (5) documented one episode of bacteremia in 55 patients undergoing ERCP.

Manipulation of a calculus through a $\mathrm{T}$ tube tract is more frequently complicated by bacteremia. Low et al (2) found bacteremia in 17 of 32 (53\%) of these procedures. Enterococcus species represented $22 \%$ of isolates, while $28 \%$ were $E s$ cherichia coli. The majority of remaining isolates were other enterobacteriaceae and aerobic Grampositive cocci (2). Thus, although unproven, the percutaneous extraction of a calculus through the T tube tract is the probable source of this patient's valve infection.

The effective treatment of this patient was hampered by a reported penicillin allergy. The infecting organism exhibited in vitro tolerance to vancomycin, and one can only speculate whether this played a role in the patient's inadequate response to this agent. It is possible a more immediate microbiological response would have been obtained had an aminoglycoside been added to vancomycin at the start of therapy. In a recent 
report, vancomycin alone was bacteriostatic for five isolates of enterococci causing endocarditis, but bactericidal for only one isolate, while a vancomycin-aminoglycoside combination was bactericidal for all but one isolate of Enterococcus faecium (6). In the patient the presenting bacteremia was cleared only after switching therapy to ampicillin and gentamicin. The effectiveness of this combination against the isolate was supported by the serum cidal titre of $1 / 256$.

An on-line computer search of the literature did not reveal any previous reports of endocarditis secondary to either ERCP or percutaneous manipulation of a biliary calculus through a $\mathrm{T}$ tube tract. The authors believe this is the first report of such a case.

\section{REFERENCES}

1. Brandenburg RO, Giuliani ER, Wilson WR, Geraci JE. Infective endocarditis - A 25 year overview of diagnosis and therapy. J Am Coll Cardiol 1983; 1:290-1.

2. Low DE, Shoenut JP, Durbahn G, et al. Bacteremia associated with percutaneous extraction of biliary tract stones. J Infect Dis 1989;159:984-8.

3. Low DE, Shoenut JP, Kennedy JK, Harding GKM, Boer BD, Micflikier AB. Risk of bacteremia with endoscopic sphincterotomy. Can J Surg 1987;30:42 1-2.

4. Low DE, Micflikier AB, Kennedy JK, Stiver HG.
Prophylaxis with antibiotics is not currently recommended for either ERCP or percutaneous extraction of biliary calculi in patients with normal heart valves, and a single case of endocarditis is not sufficient to alter this standard of practice. However, the high incidence of bacteremia during the latter procedure may be reason to evaluate this policy further. In persons with abnormal heart valves who are undergoing similar procedures, antibacterial prophylaxis should be used, although there is no proof to date that this is an effective or necessary intervention. In addition, when penicillins are contraindicated, it would seem prudent to include an aminoglycoside along with vancomycin as initial therapy for $E$ faecalis endocarditis.

Infectious complications of endoscopic retrograde cholangiopancreatography. Arch Intern Med 1980; 140:1076-7.

5. Dutta SK, Cox M, Williams RB, Wisenstat TE, Standiford HC. Prospective evaluation of the risk of bacteremia and the role of antibiotics in ERCP. J Clin Gastroenterol 1983;5:325-9.

6. Besnier JM, Leport C, Bure A, Vilde JL. Vancomycin aminoglycoside combinations in therapy of endocarditis caused by Enterococcus species and Streptococcus bovis. Eur J Clin Microbiol Infect Dis 1990;9:130-3. 


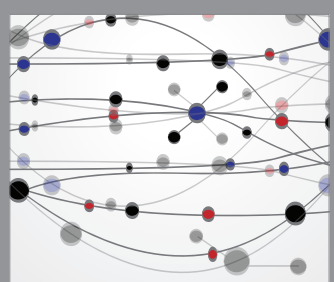

The Scientific World Journal
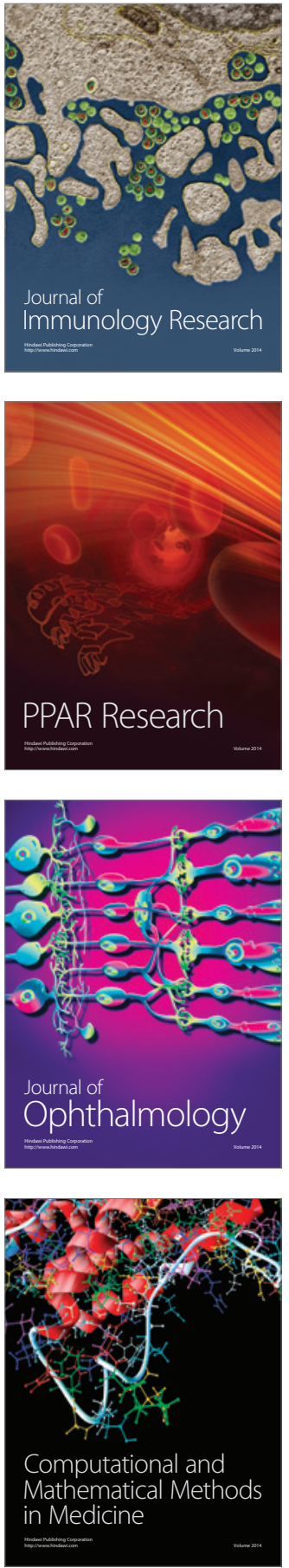

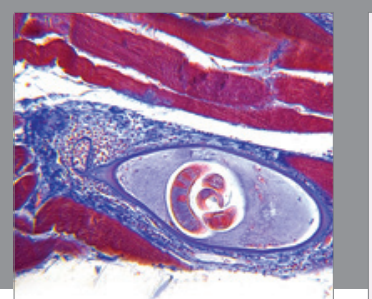

Gastroenterology Research and Practice

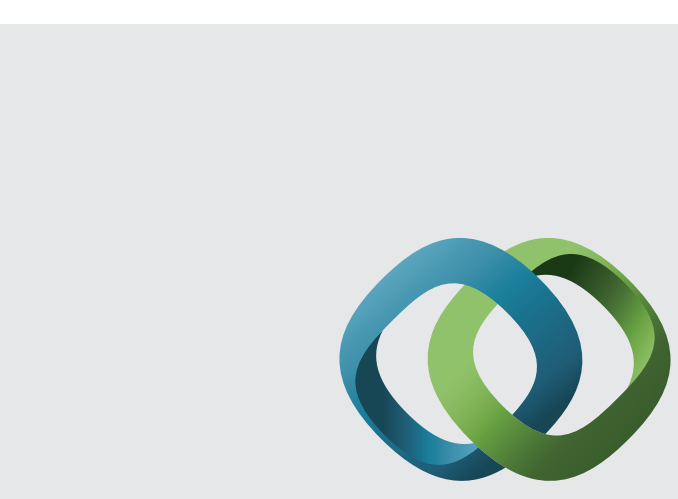

\section{Hindawi}

Submit your manuscripts at

http://www.hindawi.com
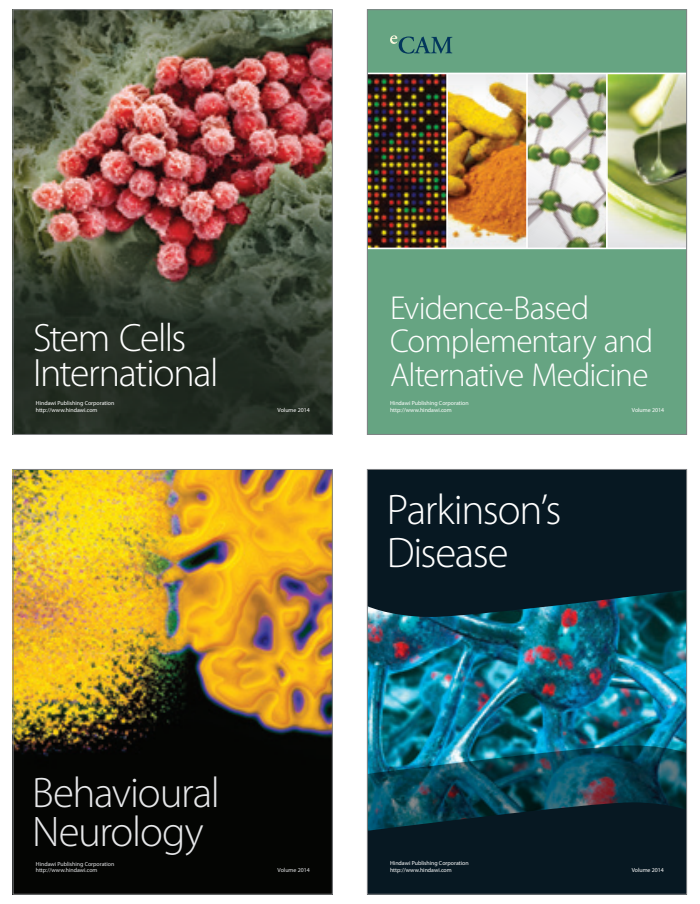
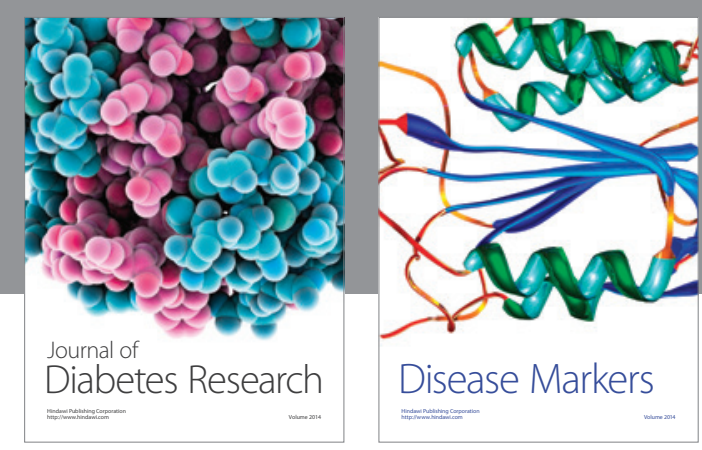

Disease Markers
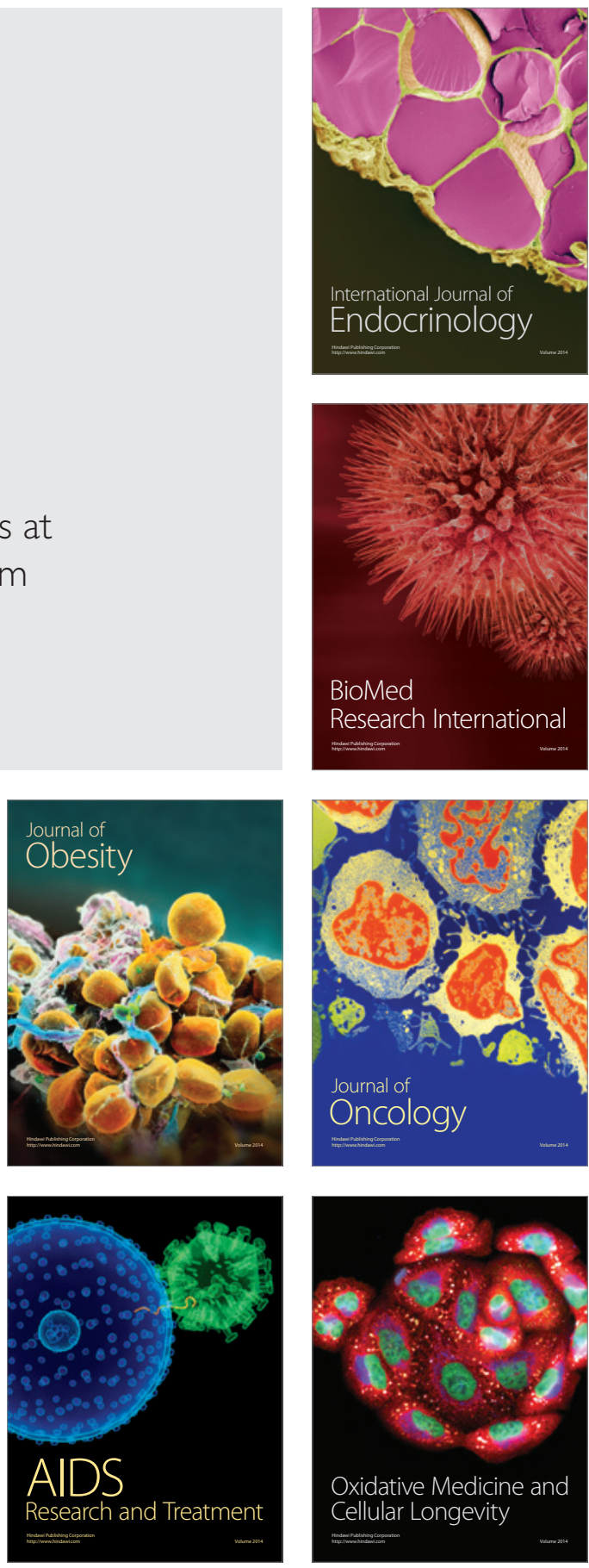